\title{
Reprogramming mRNA Expression in Response to Defect in RNA Polymerase III Assembly in the Yeast Saccharomyces cerevisiae
}

\author{
Izabela Rudzińska ${ }^{1}$, Małgorzata Cieśla ${ }^{1}$, Tomasz W. Turowski ${ }^{1,2}{ }^{2}$ Alicja Armatowska ${ }^{1}$, Ewa Leśniewska ${ }^{1}$ \\ and Magdalena Boguta ${ }^{1, * \mathbb{D}}$ \\ 1 Laboratory of tRNA Transcription, Institute of Biochemistry and Biophysics, Polish Academy of Sciences, \\ Pawińskiego 5A, 02-106 Warsaw, Poland; irudzinska@ibb.waw.pl (I.R.); goges@ibb.waw.pl (M.C.); \\ twturowski@gmail.com (T.W.T.); a.armatowska@ibb.waw.pl (A.A.); EWA_MAK@interia.pl (E.L.) \\ 2 Wellcome Centre for Cell Biology, The University of Edinburgh, Edinburgh EH8 9YL, UK \\ * Correspondence: magda@ibb.waw.pl; Tel.: +48-22-592-1322; Fax: +48-22-592-2190
}

check for

updates

Citation: Rudzińska, I.; Cieśla, M.; Turowski, T.W.; Armatowska, A.; Leśniewska, E.; Boguta, M.

Reprogramming mRNA Expression in Response to Defect in RNA Polymerase III Assembly in the Yeast Saccharomyces cerevisiae. Int. J. Mol. Sci. 2021, 22, 7298. https://doi.org/ $10.3390 /$ ijms 22147298

Academic Editor: Maurizio Ciani

Received: 31 May 2021

Accepted: 3 July 2021

Published: 7 July 2021

Publisher's Note: MDPI stays neutral with regard to jurisdictional claims in published maps and institutional affiliations.

Copyright: (c) 2021 by the authors. Licensee MDPI, Basel, Switzerland. This article is an open access article distributed under the terms and conditions of the Creative Commons Attribution (CC BY) license (https:/ / creativecommons.org/licenses/by/ $4.0 /)$.

\begin{abstract}
The coordinated transcription of the genome is the fundamental mechanism in molecular biology. Transcription in eukaryotes is carried out by three main RNA polymerases: Pol I, II, and III. One basic problem is how a decrease in tRNA levels, by downregulating Pol III efficiency, influences the expression pattern of protein-coding genes. The purpose of this study was to determine the mRNA levels in the yeast mutant rpc128-1007 and its overdose suppressors, RBS1 and PRT1. The rpc128-1007 mutant prevents assembly of the Pol III complex and functionally mimics similar mutations in human Pol III, which cause hypomyelinating leukodystrophies. We applied RNAseq followed by the hierarchical clustering of our complete RNA-seq transcriptome and functional analysis of genes from the clusters. mRNA upregulation in rpc128-1007 cells was generally stronger than downregulation. The observed induction of mRNA expression was mostly indirect and resulted from the derepression of general transcription factor Gcn4, differently modulated by suppressor genes. rpc128-1007 mutation, regardless of the presence of suppressors, also resulted in a weak increase in the expression of ribosome biogenesis genes. mRNA genes that were downregulated by the reduction of Pol III assembly comprise the proteasome complex. In summary, our results provide the regulatory links affected by Pol III assembly that contribute differently to cellular fitness.
\end{abstract}

Keywords: yeast; gene expression; RNA polymerase

\section{Introduction}

Transcription in a eukaryotic cell is carried out by three RNA polymerases: Pol I, II, and III. The set of transcripts synthesized by Pol II is extremely complex because it includes thousands of different protein-coding mRNAs. In contrast, Pol I and Pol III are more specialized. Pol I is responsible for the synthesis of rRNA, and Pol III is responsible for the synthesis of tRNA and 5S rRNA, which are fundamental for the process of translation. One basic problem is how the decrease in tRNA levels caused by downregulating Pol III influences mRNA synthesis by Pol II. To address this, we used yeast mutant rpc128-1007 located in the Rpc128 catalytic subunit near the contact points for the association between Rpc128 and the Rpc40-Rpc19 heterodimer [1]. Since the formation of Rpc128-Rpc40-Rpc19Rpb12-Rpb10 subcomplex is the critical step in Pol III biogenesis [2,3], the rpc128-1007 mutation has severe consequences for the assembly of the active Pol III complex and hence the ability of the cell to support Pol III transcription activity [1]. Recently published structures of human Pol III revealed a disease-relevant cluster, which is functionally similar to the yeast mutant rpc128-1007 [4-6]. The mutations lead to neurodegenerative disorders called hypomyelinating leukodystrophies, and many are predicted to affect the interface between Pol III subunits, especially affecting assembly with the assembly platform, homologous to Rpc40-Rpc19-Rpb12-Rpb10 heterotetramer. Using the rpc128-1007 mutant and a 
yeast model we can therefore predict how global mRNA expression will be changed by hypomyelinating leukodystrophies.

In the yeast model, genes that can suppress the cold-sensitive phenotype of the rpc1281007 mutant encoded the Rpb10 subunit, which is shared by all three RNA polymerases and participates in the assembly of Pol III and Pol I [7], and the fructose bisphosphate aldolase Fba1, which is involved in the control of Pol III-directed transcription [8]. The growth phenotype of the rpc128-1007 mutant was also suppressed by the overproduction of Rbs1 and Prt1 proteins [1].

The molecular mechanism of suppression by Rbs1 relies on the improvement of Pol III assembly in rpc128-1007 cells. Rbs1 stimulates biogenesis of the Pol III complex both directly and indirectly. First, it binds to the Pol III complex or subcomplex and facilitates its translocation to the nucleus. Following dissociation from Pol III in the nucleus, Rbs1 is exported back to the cytoplasm by Crm1 exportin [1]. Second, Rbs1 in the cytoplasm binds to a subset of mRNA and interacts with its regulatory sequences. The interaction between $\mathrm{Rbs} 1$ and mRNA depends on the $\mathrm{R} 3 \mathrm{H}$ domain in the Rbs1 sequence. The binding of Rbs1 to the $3^{\prime}$-untranslated region (UTR) in RPB10 mRNA leads to an increase in the synthesis of Rpb10 protein, thereby indirectly stimulating Pol III assembly [2,9].

Prt1/eIF3b is one of five subunits of the translation initiation factor eIF3 [10]. Yeast eIF3 serves as an essential scaffold that promotes the binding of other initiation factors to the $40 \mathrm{~S}$ ribosomal subunit, where it coordinates their actions during translation initiation [11]. Considering the basic function of Prt1 in the control of translation initiation, its role in Pol III assembly appears unlikely, thus suggesting an indirect mechanism of $r p c 128-1007$ suppression by Prt1.

The main aim of the work was to provide a global view of a regulatory network that involves the coordination of Pol III assembly and Pol II activity. Using RNA sequencing (RNA-seq) analysis, we explored the way in which mRNA transcription by Pol II is influenced by the rpc128-1007 mutant and whether the observed changes can be overcome by an overdose of the suppressor genes RBS1 and PRT1. The reduction in Pol III assembly resulted in the extensive reprogramming of yeast Pol II genes, which are clustered in the functional classes. Expression of selected genes, examined by RT-qPCR, was specifically affected in the rpc128-1007 mutant and suppressor strains.

\section{Results}

2.1. Induction of GCN4-lacZ Reporter in the Pol III Assembly Mutant Is Differentially Affected by the Suppressors RBS1 and PRT1

The rpc128-1007 mutation has severe consequences for the assembly of the active Pol III complex and hence for the capacity of the cell to support transcription activity and growth. Reduced viability of $r p c 128-1007$ cells was correlated with a declined synthesis of Pol III transcripts [1]. We reasoned that a decrease in tRNA levels in rpc128-1007 cells might result in a rearrangement of mRNA expression. A major effect that was previously observed in other mutants with Pol III machinery defects was the activation of Gcn4 transcription [12]. This regulatory response, known as general amino acid control (GAAC), relies on the translational induction of GCN4 mRNA [13].

Hence, we investigated whether a defect in Pol III assembly similarly results in elevated Gcn4 translation. We utilized a widely employed CEN-based reporter plasmid carrying a GCN4 promoter and 5'UTR translation regulatory sequences. Driving the expression of a lacZ gene provided a quantitative measure for Gcn 4 translation by assessing $\beta$-galactosidase activity [12].

More than a six-fold induction of GCN4-lacZ expression was measured in the rpc1281007 cells with respect to the wild type under nonstarvation conditions (Figure 1A), indicating that GCN4 translational induction was constitutively operating in the mutant with the Pol III-assembly defect. As expected, a further increase in GCN4-lacZ expression was observed when the cells were starved for amino acids for $3 \mathrm{~h}$. These measurements are consistent with the results of Conesa et al., 2005 [12], who observed induction of GCN4 in the majority of Pol III mutants. 


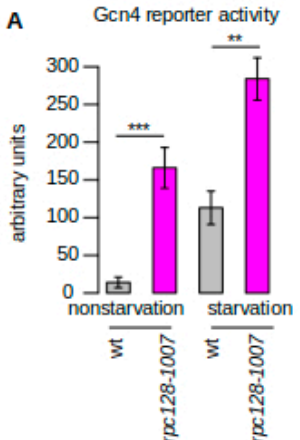

C

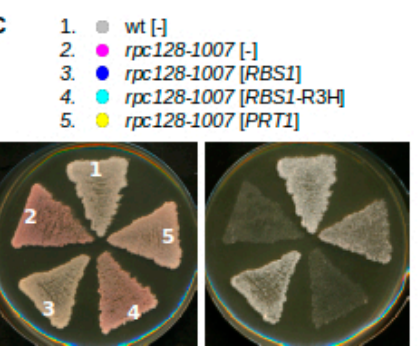

YPD $30^{\circ} \mathrm{C}$ YPD $16^{\circ} \mathrm{C}$

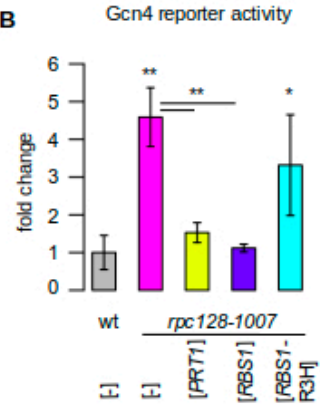

D
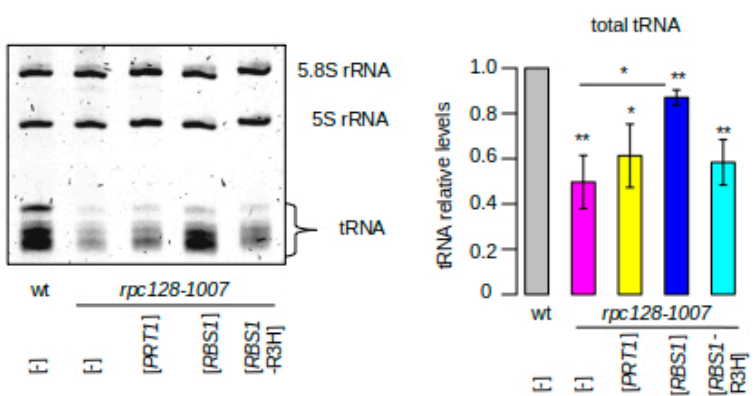

E
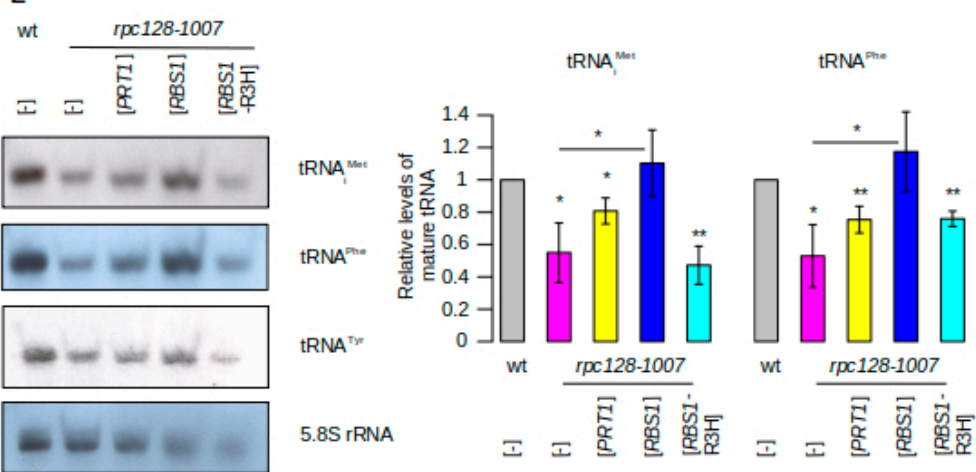

tRNA

tRNA $^{\text {Th }}$

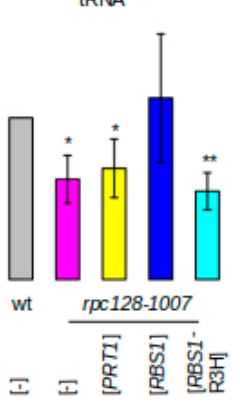

Figure 1. RBS1 and PRT1 as overdose suppressors of the Pol III assembly mutant rpc128-1007. (A) Expression of the GCN4-lacZ in rpc128-1007 mutant. The rpc128-1007 mutant and isogenic wild-type strains, transformed with p180 plasmid (GCN4-lacZ, CEN, URA3), were grown under nonstarvation or starvation conditions, as described in the Materials and Methods section. Extracts were prepared and assayed for $\beta$-galactosidase activity (expressed as nanomoles of $o$-nitrophenol$\beta$-D-galactopyranoside hydrolyzed per minute per microgram of total protein). The reported values are averages of three independent measurements (number of biological replicates $n=3$ ). (B-D) The rpc128-1007 mutant was transformed with a control empty vector [-] or multicopy plasmids [PRT1], [RBS1], and [RBS1-R3H]. (B) The effect of suppressors on GCN4-lacZ expression. Cells harboring p180 plasmid (GCN4-lacZ, CEN, URA3) were grown in YPD, harvested in log phase. $\beta$-galactosidase activity was determined and calculated relative to the amounts in the wt strain, which was set as $1(n=3)$. (C) Suppression of the rpc128-1007 growth phenotype. Cells that were grown on a YPD plate were replicated on YPD plates and incubated for three days at the respective temperatures. (D) Determination of total tRNA levels. Small RNA species were isolated and separated on a $7 \mathrm{M}$ urea- $6 \%$ polyacrylamide gel using equal amounts of RNA per lane $(2.5 \mu \mathrm{g})$ and stained with ethidium bromide $(n=3)$. (E) Determination of specific tRNA levels by northern hybridization with probes specific for mature $\mathrm{tRNA}_{\mathrm{i}}{ }^{\mathrm{Met}}, \mathrm{tRNA}^{\text {Phe }}$, and $\mathrm{tRNA}{ }^{\mathrm{Ty}}$. $\mathrm{tRNA}$ amounts in D and $\mathrm{E}$ were normalized to the loading control (5.8 S rRNA) and calculated relative to amounts in the wt strain, which was set as 1 . Bars represent the mean \pm standard deviation (SD) of three independent experiments $(n=3)$. Values of $p$ were calculated using a two-tailed paired $t$-test: ${ }^{*} p<0.05$; ${ }^{* *} p<0.01$; ${ }^{* * *} p<0.001$. Asterisks just under bars show the $p$-value in comparison to the level in wt; other comparisons are annotated by lines. To clarify the plots, bars are colored as follows: rpc128-1007 with control vector-pink; with [PRT1], [RBS1], and [RBS1-R3H] -yellow, dark blue, and light blue, respectively; grey was used for control strains. The same color code and calculation of $p$-values are used in the figures hereafter. 
To more comprehensively investigate the connection between Pol III biogenesis and GCN4 induction, we extended our studies on the rpc128-1007 mutant and its overdose suppressors. Two suppressor genes, RBS1 and PRT1, were considered, the overdose of which complemented the cold-sensitive phenotype of the rpc128-1007 mutant. The RBS1 gene encodes the RNA-binding protein, which stimulates Pol III biogenesis; whereas the product of the PRT1 gene is a subunit of the translation initiation factor, eIF3. In general, genetic suppression by RBS1 and PRT1 correlated with a decrease in GCN4 induction in the rpc128-1007 mutant to a level close to that of the wild type (Figure 1B,C). When the rpc128-1007 cells were transformed by the plasmid that carried the RBS1-R3H mutant allele, in which the conserved R57 and H61 residues that are located within the RNAbinding domain $\mathrm{R} 3 \mathrm{H}$ were changed to alanine, neither genetic suppression nor a significant decrease in GCN4 expression was observed (Figure 1B,C, strain 4).

We supposed that the reprogramming of GCN4 expression in the rpc128-1007 mutant and suppressor strains is related to the levels of tRNA, especially tRNA ${ }_{i}{ }^{\text {Met. }}$. The $r p c 128$ 1007 strain carried additional SUP11 and ade1-2 mutations, which allowed us to monitor the tRNA-dependent phenotype according to the colony color. This was possible because the presence of the ade2-1 nonsense mutation led to pigment accumulation when the dosage of the suppressor tRNA SUP11 (Tyr/UAA) was low. The low SUP11 dosage and the resulting red colony color were presumed to indicate that the global low tRNA levels were a consequence of a decrease in Pol III transcription in the rpc128-1007 cells (Figure 1C, strain 2). Complementation by RBS1 and PRT1 resulted in a white and light pink colony, respectively (Figure 1C, strains 3 and 5), suggesting that the suppression of $r p c 128-1007$ due to an overdose of $P R T 1$ is relatively weak.

To examine the tRNA levels, small RNA species were separated on a urea-polyacrylamide gel using equal amounts of RNA per lane and stained with ethidium bromide. The level of 5.8S rRNA was comparable in rpc128-1007 and wild-type cells and thus served as an internal control. Although $5 \mathrm{~S}$ rRNA is also a product of Pol III, its levels are unaffected in rpc128-1007 cells. This is unsurprising because many other Pol III mutants lead to a decrease in tRNA synthesis but do not alter the transcription of $5 S$ rRNA [14]. As expected, tRNA levels were low in the $r p c 128-1007$ mutant that carried an empty vector or plasmid with a mutated RBS1-R3H allele and were increased by an overdose of native RBS1 (Figure 1D). This is consistent with the $\mathrm{R} 3 \mathrm{H}$ domain-dependent role of Rbs1 in improving Pol III assembly and the recovery of tRNA synthesis in rpc128-1007 cells [2]. Global tRNA levels in rpc128-1007 cells were not significantly changed by PRT1 overexpression. This could imply that PRT1 does not act by restoring Pol III assembly.

The same cells were examined by northern blot to quantify the amounts of single tRNAs. Levels of three representative tRNAs: $\mathrm{tRNA}_{i}{ }^{\mathrm{Met}}$, $\mathrm{tRNA}^{\text {Phe }}$ and $\mathrm{tRNA}{ }^{\mathrm{Tyr}}$, demonstrated an approximate two-fold decrease in the rpc128-1007 mutant relative to the wild-type control (Figure 1E). RBS1 overexpression elevated tRNA levels in rpc128-1007 cells; whereas the changes due to PRT1 overexpression determined for the three specific tRNAs were not statistically significant as compared to levels in the rpc128-1007 mutant (Figure 1E).

RBS1 overexpression elevated tRNA levels in rpc128-1007 cells; whereas the changes due to PRT1 overexpression determined for the three specific tRNAs were not statistically significant (Figure 1E). In summary, rpc128-1007 suppression by RBS1 was correlated with the decreased activity of GCN4 reporter and increased tRNA levels; whereas we did not observe a full correlation for suppression by PRT1. This difference motivated us to analyze the transcriptional response of rpc128-1007 and suppressor mutants on a genome-wide scale.

\subsection{RNA-seq Analysis Reveals Distinct Gene Expression Signatures of the rpc128-1007 Mutant and Its Suppressors}

To compare the effects of $r p c 128-1007$ and its suppressors on overall Pol II-dependent gene expression, we performed RNA-seq analysis. mRNA was isolated from the rpc1281007 mutant that was optionally transformed with PRT1, RBS1, and RBS1-R3H plasmids. The same mutant that was complemented by the native RPC128 gene, expressed from 
the centromeric plasmid, was used as an isogenic wild-type control (referred to as wt). Cells were grown overnight on selective medium to prevent plasmid loss, transferred to a nonselective glucose-rich medium, grown to the exponential phase, and harvested. The results of the RNA-seq experiments, performed with three biological repetitions, were subjected to differential expression analysis using the DEseq 2 method [15-17]. The complete data of the RNA-seq analysis are presented in Supplementary Table S2.

We first examined the overall similarity between samples by principal component analysis (PCA) (Figure 2A) and hierarchical clustering (Figure S2A) for protein-coding genes. Three independent biological replicates from the same strain were clustered together, confirming data reproducibility. The analysis revealed two separate groups of strains that were genetically close: the first included the wt and the two suppressor strains $r p c 128$ 1007 [PRT1] and rpc128-1007 [RBS1]; the second included rpc128-1007 and the inactive suppressor rpc128-1007 [RBS1-R3H] (Figure 2A). These results correlated with the results of genetic suppression, as determined by growth under restrictive conditions. which was observed for strains from the first group but not the second group (Figure 1C).

To categorize genes that are involved in the suppression of the rpc128-1007 phenotype, we performed hierarchical clustering of our complete RNA-seq transcriptome (Figure 2B). All genes without a significant change $(p \geq 0.05)$ in expression were annotated as unchanged and $\log 2$ of fold change was replaced with zero $(n=3557$; white color on the heatmap). Transcription upregulation was generally stronger, exceeding a four-fold change (two on the logarithmic scale), and involved more genes than downregulation, which was characterized by a two-fold change. Groups of up- and downregulated genes, indicated by green and red, respectively, clustered together. Generally, the rpc128-1007 and rpc128-1007 [RBS1-R3H] expression profiles were similar, which is consistent with [RBS1-R3H] having no effect on phenotypic suppression (Figure 1C, Supplementary Figure S1) and a minimal difference between both strains, as confirmed by PCA (Figure 2A).

To elucidate differences between rpc128-1007 and the suppressed mutants rpc128-1007 [RBS1] and rpc128-1007 [PRT1], differentially expressed genes were clustered by expression pattern similarity between the examined strains (Figure 2C). For clustering, we only selected genes with at least one differentially expressed sample as compared to wt $(n=3023)$, and divided them into 10 clusters using a k-mean clustering algorithm. The suppressors rpc1281007 [PRT1] and $r p c 128-1007$ [RBS1] were clearly different from the rpc128-1007 mutant strain, but they were also different from each other. These differences are consistent with their distinct effects on tRNA expression (Figure 1D,E) and, together with the phenotypic differences (Figure 1C), indicate different mechanisms of rpc128-1007 suppression.

We analyzed genes from all clusters using annotations in the String Database (https:/ / string-db.org/, accessed on 31 May 2021). Two top GO term functional categories for each cluster are presented in Supplementary Figure S2B. To validate the most interesting findings, we used an independent approach, in which the entire gene category was selected, verified using a statistical approach, and presented as boxplots (Figure 2D, Supplementary Figure S2C).

Genes in clusters 1 and 2 exhibited an increase in expression in the rpc128-1007 strain (Figure 2C), which was generally compromised in the suppressor strains. Among these genes, we identified strong enrichment for amino acid biosynthesis genes (Figure S2B), which are targets of the Gcn 4 transcription factor. In fact, most genes from clusters 1 and 2 were under the control of Gcn4, which was noticed using the annotation of Gcn4 response genes from the published data $(n=511)[18,19]$. Gcn4-dependent genes were upregulated in the rpc128-1007 mutant (Figure 2D, $p=3.5 \times 10^{-40}$ ), and suppression by either PRT1 or RBS1 overdose correlated with a significant decrease in their expression $\left(p=3.5 \times 10^{-47}\right.$ and $\left.1.6 \times 10^{-25}\right)$. For PRT1 overdose, the expression of Gcn4-dependent genes was even lower than in the wt strain $\left(p=6 \times 10^{-9}\right)$. Another intriguing observation was the effect of the mutated [RBS1-R3H] plasmid, which was used as a negative control. The overproduction of mutated Rbs1-R3H in rpc128-1007 cells led to an increase in the expression of genes in clusters 1 and 2, which are mostly Gcn4-dependent (Figure 2D,E). 


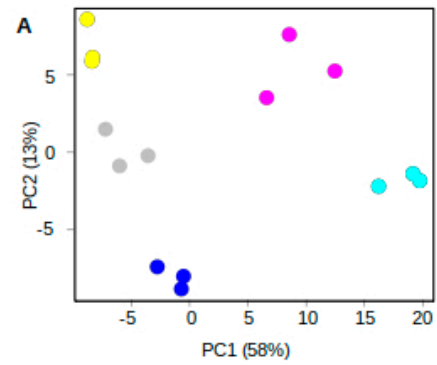

B
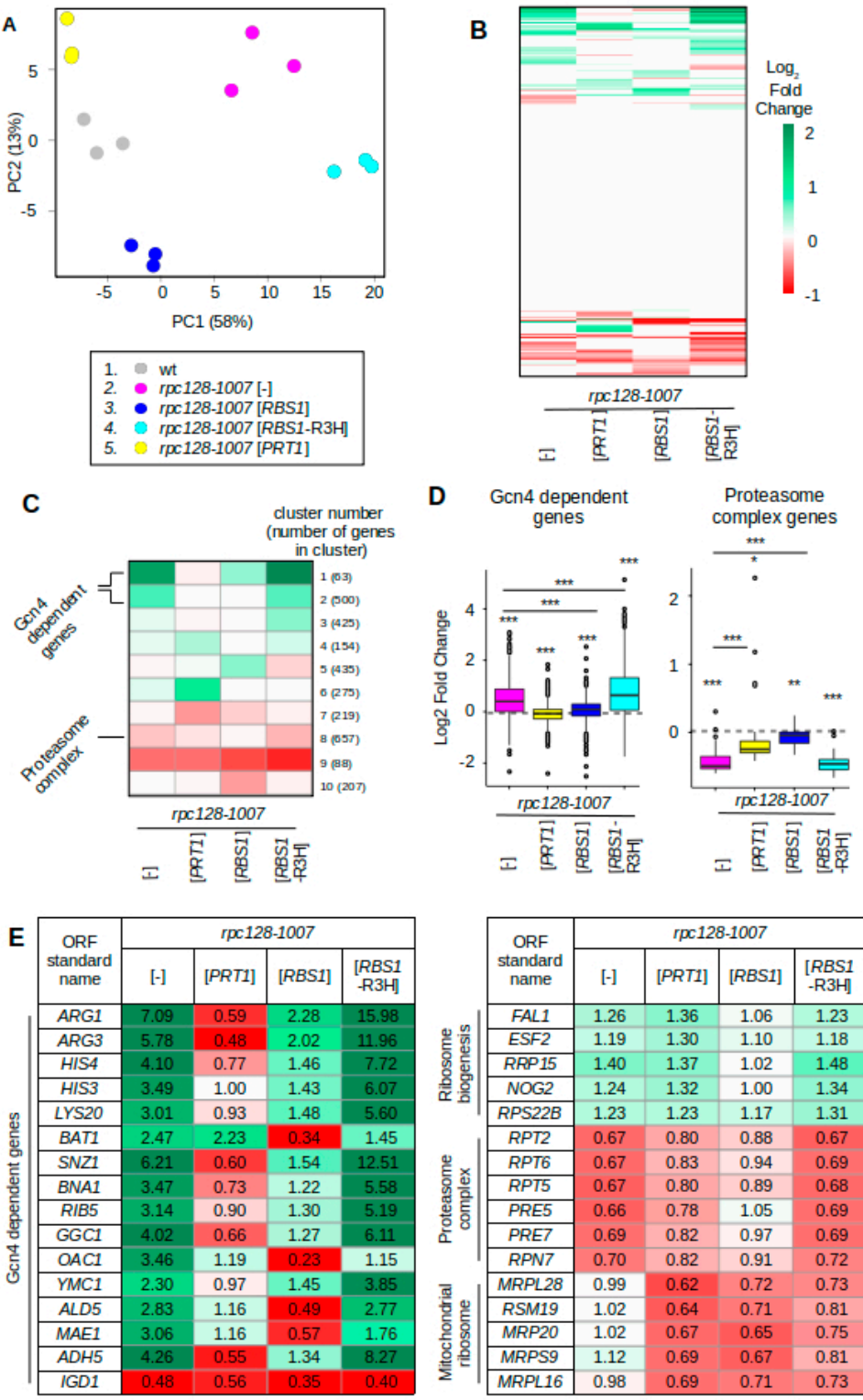

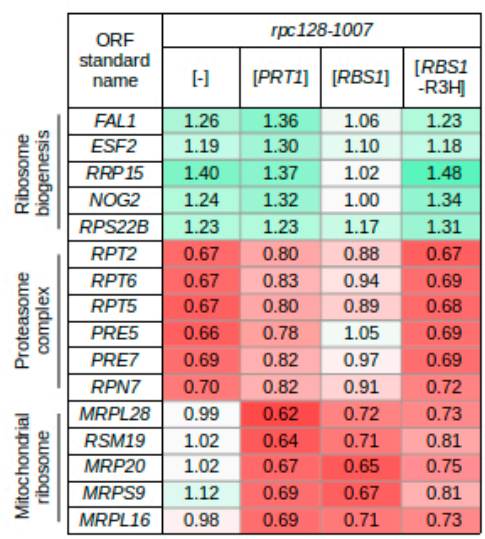

Figure 2. RNA-seq analysis reveals a common mechanism of $r p c 128-1007$ suppression by RBS1 and PRT1 despite functional differences. The rpc128-1007 mutant strains were transformed with [RPC128], empty vector, or overexpressing [PRT1], [RBS1], or [RBS1-R3H] and subjected to RNA-seq analysis $(n=3)$. (A) Principal component analysis (PCA) of the RNA-seq data shows differences between strains and a good level of reproducibility. The axis titles show the extent of variation, which is explained by a given principal component. (B) Heatmap of hierarchical clustering between RNA-seq samples based on the entire yeast transcriptome. Data are shown as $\log 2$ of the ratio between each strain and wt. (C) Heatmap of the clustering analysis of differentially expressed genes. Selected enriched functional categories are annotated. (D) Boxplots of changes in mRNA levels for Gcn4dependent genes (left) or proteasome complex genes (right). Statistical significance was calculated using the Mann-Whitney test. ${ }^{*} p<0.05,{ }^{* *} p<0.01,{ }^{* * *} p<0.001$. Asterisks just under boxes represent $p$-value in comparison to the level in $\mathrm{wt}$; other comparisons are annotated by lines. (E) RNA-seq data for representative genes that belong to given functional categories. Data were selected from the supplemental Table S2 where the adjusted $p$-values for each expression value are specified.

An opposite trend was observed in cluster 8 , which contained functional categories that are involved in protein degradation, including 27 of the 31 subunits of the proteasome (Figure 2C,D, Supplementary Figure S2B). Genes that comprise the proteasome complex 
were downregulated in the $r p c 128-1007$ mutant (Figure 2D right panel; $p=9.6 \times 10^{-10}$ ), and the suppressors PRT1 and RBS1 led to its partial reversal $\left(p=5.9 \times 10^{-8}, p=8.9 \times 10^{-9}\right)$.

Genes that were specifically upregulated by the overexpression of $R B S 1$ are represented by cluster 5 (Figure 2C). Interestingly, these genes were generally unaffected in rpc128-1007 cells but downregulated in rpc128-1007 [RBS1-R3H] cells. In this cluster, we found genes involved in various functional categories. For example, genes involved in glucose transport were significantly upregulated by Rbs1 (Supplementary Figures S2B and S3). These effects may have been driven by the RNA-protective function of Rbs1, mediated by Rbs1 binding to $3^{\prime}$-UTR regulatory sequences in mRNAs that depend on the active R3H domain in Rbs1 [2]. However, the modest and nonsignificant enrichment of Rbs1 targets that were identified by the CRAC analysis was observed among genes in cluster 5 (data not shown).

Clusters 4 and 6 represented genes that were specifically upregulated by PRT1 overdose; however, we were unable to identify a meaningful GO category; therefore, we focused on individual genes. Among these, we found genes that encode factors that are involved in rRNA processing and biogenesis of cytoplasmic ribosomes (Supplementary Figure S2C). These effects were rather small; however, some increase in the expression of ribosome biogenesis genes was observed in all strains that harbored the $r p c 128-1007$ mutation regardless of the presence of suppressor genes (Supplementary Figure S2C left panel).

Finally, cluster 7 represented genes that likely represent specific regulation that is linked to the PRT1-mediated suppression of the rpc128-1007 mutation (Figure 2C). Interestingly, we identified genes that are involved in mitochondrial translation as a major functional group among genes from cluster 7 and this group strongly overlaps with group mitochondrial gene expression (Figure S2B). After validation, we found that those genes were downregulated by both PRT1 and RBS1 suppressors (Figure S2C, $p=2.8 \times 10^{-12}$ and $\left.1.3 \times 10^{-19}\right)$. Changes in expression for a few example genes from selected categories are given in Figure 2E.

Effect of rpc128-1007 and Suppressors on the Expression of Selected Gcn4-Dependent Genes

Mostly altered by the rpc128-1007 mutation are the Gcn4-dependent genes. Considering greater-than-two-fold changes in expression to be significant, 124 differentially expressed genes were found in the rpc128-1007 mutant, of which 98 are under Gcn 4 control (Supplementary Table S2; examples in Figure 2E). Individual Gcn4-dependent genes were, however, induced to various extents in the $r p c 128-1007$ mutant and their expression was differentially affected by PRT1 and RBS1. The genes induced in rpc128-1007 cells are involved in the biosynthetic pathways of amino acids (ARG1, ARG3, HIS4, HIS3, LYS20, BAT1), cofactors and vitamins (SNZ1, BNA1, RIB5), mitochondrial transport (GGC1, OAC1, YMC1), and metabolism ( $A L D 5, M A E 1, A D H 5)$. One example of a Gcn4-dependent gene that was downregulated in rpc128-1007 cells is IGD1, which is involved in glycogen homeostasis.

Changes in the expression of selected Gcn4-dependent genes were confirmed by direct RT-qPCR measurements (Figure 3). As expected for these genes, induction of expression was prevented by GCN4 deletion or tRNA ${ }_{i}^{\text {Met }}$ overproduction. Levels of ARG1 and ARG3 transcripts were 6.2- and 6.1-fold higher, respectively, in the rpc128-1007 mutant than in the control (wild-type strain). Overexpression of the PRT1 gene reduced ARG1 mRNA and ARG3 mRNA levels in the rpc128-1007 mutant by 9.8- and 11-fold, approaching levels that were two-times lower than in the control (wild-type strain). Suppression by RBS1 was relatively weaker, with 2.5- and 3.4-fold decreases in ARG1 mRNA and ARG3 mRNA levels being observed. The effect of the [RBS1-R3H] plasmid was the opposite, with ARG1 and ARG3 transcript levels in the rpc128-1007 mutant increasing 2.2- and 1.7-fold, respectively. These data corroborate the RNA-seq results (Figure 2D). 

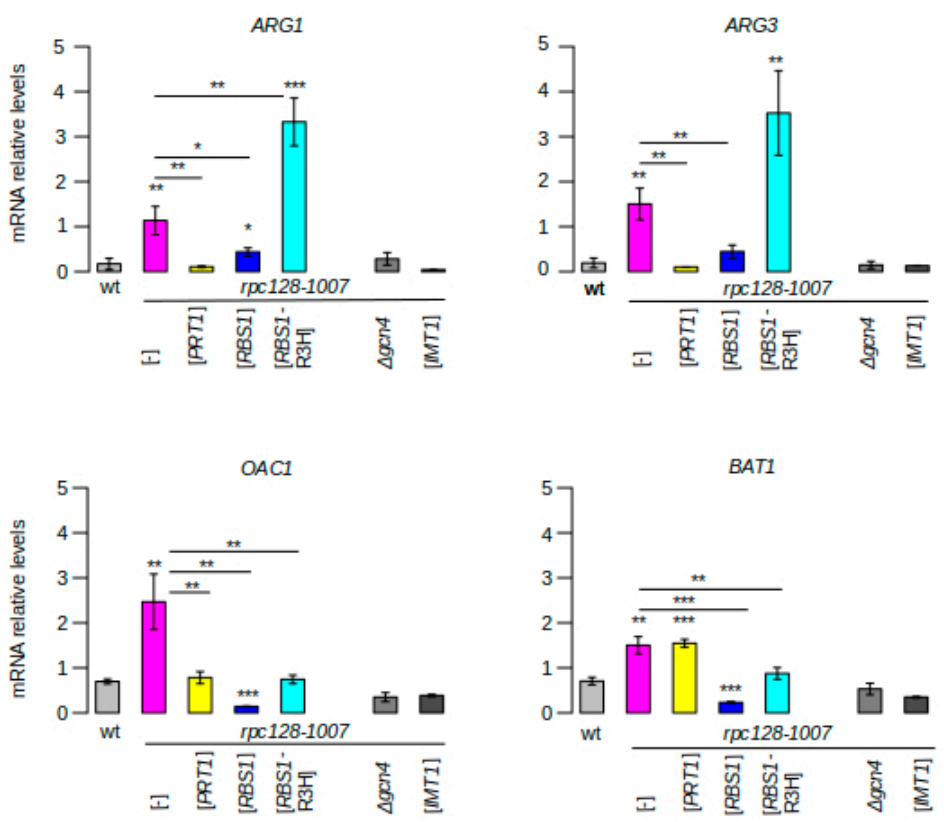

Figure 3. Induction of GCN4 expression in the Pol III assembly mutant is differentially affected by the suppressors RBS1 and PRT1. RNAs that were isolated from the rpc128-1007 mutant, which was transformed with the indicated plasmids and control strains (wt, rpc128-1007 gcn4 $\Delta$ ), were analyzed by RT-qPCR with specific probes $(n=3)$. Values of $p$ were calculated using a two-tailed paired $t$-test: ${ }^{*} p<0.05 ;{ }^{* *} p<0.01 ;{ }^{* * *} p<0.001$.

However, for certain Gcn4-dependent genes in which induction was relatively weak in the rpc128-1007 mutant, the suppression by RBS1 was stronger than suppression by PRT1. This minor class of genes is represented by OAC1, MAE1, and ALD5 (Figure 2E). For example, the induction of $O A C 1$ in the rpc128-1007 mutant, which was more than a three-fold, was decreased to the level of 0.07 by RBS1 (i.e., a 15 -fold decrease) and 0.34 by PRT1 (i.e., a 2.9-fold decrease). Moreover, no effect of $P R T 1$ was observed as regards the induction of BAT1 expression, which was more than two-fold (Figure 3). Interestingly, the [RBS1-R3H] plasmid that encoded Rbs1 with an inactive $\mathrm{R} 3 \mathrm{H}$ domain had no effect on the expression of these genes in rpc128-1007 cells (Figure 2E). Altogether, while the examined genes belong to one functional category, they might be differently affected by PRT1 and RBS1 suppressors.

\section{Discussion}

The interplay between the three nuclear RNA polymerase systems is presumably a key aspect of growth control. Pol III synthesizes very abundant and essential RNAs whose levels might influence the expression patterns of protein-coding genes. Remarkably, Pol II is preferentially recruited in close proximity to tRNA genes that display high Pol III occupancy [20-25]. The two main aspects of the influence of RNA Pol III-directed transcription on the expression of RNA Pol II-transcribed genes in S. cerevisiae were studied. First, there is a positional effect that is exerted by an extremely high rate of transcription and the occupancy of Pol III machinery on neighboring Pol II genes and vice versa. The positional effect of Pol III on mRNA expression, however, only operates to a limited extent [26]; whereas Pol II transcription robustly interferes with Pol III function at specific tRNA genes [27]. Second, there is a genome-wide effect of Pol III deficiency on the expression of Pol II genes. This was revealed by microarray analyses of mutants in different components of RNA Pol III transcription machinery and of single tRNA gene deletions mutants [12,28]. Deletions of tRNA genes from single-copy families elicited a stress response; whereas deletions of genes from multicopy families led to increased expression of genes involved in translation. 
Other functional groups of mRNA were differentially affected by deletions of tRNA genes from single- and multicopy families [28].

In the present study, we used RNA-seq to analyze the expression of Pol II genes in the Pol III assembly mutant rpc128-1007 and this study was extended to the overdose suppressors, which overcome the cold-sensitive phenotype of $r p c 128-1007$. The suppressor genes, RBS1 and PRT1, account for Pol III assembly and translation initiation, respectively.

The rpc128-1007 mutant results in severe consequences for the activity of Pol III and leads to a decrease in global tRNA levels, which involved a two-fold decrease in the amount of $\mathrm{tRNA}_{\mathrm{i}} \mathrm{Met}^{\text {Met }}$ (Figure 1). The suppression of the Pol III assembly defect by Rbs1 overdose led to a back increase in both global tRNA and tRNA ${ }_{i}{ }^{\text {Met }}$ levels. Although PRT1 overexpression clearly overcame the growth phenotype of the rpc128-1007 mutant, the correlation with the increase in tRNA levels was less prominent (compare Figures 1C and 1D,E) and, therefore, functional suppression of the assembly defect by PRT1 could be indirect. The decrease in tRNA levels in the rpc128-1007 mutant led to a genome-wide Gcn4 response, which was differentially affected by an overdose of PRT1 and RBS1, indicating that $r p c 128-1007$ suppression downregulates GCN4 but occurs through two different molecular mechanisms.

Gcn4 is a transcription factor that is important for activating amino acid biosynthetic genes in response to amino acid starvation (reviewed in [29]) and regulating diverse cellular processes, including purine biosynthesis, autophagy, organelle biosynthesis, the endoplasmic reticulum stress response, and the induction of mitochondrial transport carrier proteins $[18,30,31]$. Gcn 4 protein levels are primarily determined by translation initiation and protein degradation rather than by transcription. In yeast, the Gcn4 response relies on the derepression of GCN4 mRNA translation and depends on the rate of ternary complex (TC) binding to $40 \mathrm{~S}$ ribosomes. During translation initiation, methionyl-tRNA $\mathrm{A}_{\mathrm{i}}{ }^{\mathrm{Met}}$ is recruited in a TC with eIF2 and GTP to the 40S subunit. This is followed by the recruitment of mRNA to form the $48 \mathrm{~S}$ preinitiation complex, which scans mRNA for AUG recognition. The translation of Gcn 4 is regulated by four small upstream open reading frames (uORFs) in the $5^{\prime}$ leader region of GCN4 mRNA. GCN4 translation is normally repressed because ribosomes dissociate from the mRNA after translation of the fourth inhibitory uORF. However, the decrease in TC concentration makes it more likely that a scanning ribosome will scan through the fourth $\mathrm{UORF}$ and then efficiently initiate translation on the GCN4 sequence. In yeast that were starved of amino acids, the formation of TC is indirectly inhibited by Gen2 kinase, which phosphorylates eIF2 and converts it to the inactive form [29]. The concentration of TC can also be decreased by the direct depletion of initiator tRNA ${ }_{i}{ }^{\text {Met }}$ in mutants with defects in tRNA synthesis, maturation, or nuclear export [12,32-34], and in a mutant with RPL20B deletion [35]. These mutants also derepress GCN4 translation under nonstarvation conditions and belong to the Gcd- (general control derepressed) category.

In the rpc128-1007 mutant that was grown under nonstarvation conditions, we observed up to a 10-fold increase in the induction of Gcn4-dependent genes that are involved in the synthesis of amino acids (ARG1, ARG3, HIS4, HIS3, LYS20, BAT1), cofactors and vitamins (SNZ1, BNA1, RIB5), mitochondrial transport (GGC1, OAC1, YMC1), and metabolism (ALD5, MAE1, ADH5). Therefore, the rpc128-1007 mutant had a Gcd- phenotype. The suppression of this Gcd- phenotype by RBS1 overdose was consistent with the enhancement of Pol III assembly and the back increase in both global tRNA and tRNA $\mathrm{A}_{\mathrm{i}}^{\text {Met }}$ levels in rpc128-1007 cells (Figure 1D,E). One interesting observation was the suppression of the Gcdphenotype by PRT1 overexpression in the rpc128-1007 mutant (Figure 1B, Figure 3). This effect was not apparently correlated with $\mathrm{tRNA}_{\mathrm{i}}{ }^{\text {Met }}$ levels, suggesting that the mechanism of rpc128-1007 suppression by PRT1 is different from the simple correction of the Pol III assembly defect.

Prt1/eIF3b is one of the five subunits of the translation initiation factor eIF3 [10]. eIF3 stimulates the binding of TC and mRNA to 40S subunits [36]. eIF3 also has critical functions downstream of the $48 \mathrm{~S}$ complex assembly and prior to $60 \mathrm{~S}$ subunit joining, which impacts ribosomal scanning on GCN4 mRNA and AUG selection [37]. This suggests that 
Prt1 overdose is sufficient for the derepression of GCN4 translation in the rpc128-1007 mutant, with no apparent changes in $\mathrm{RNAA}_{\mathrm{i}}{ }^{\text {Met }}$ abundance. Therefore, Prt1-mediated suppression was expected to result from the prevention of rescanning ribosomes to bypass uORFs when TC levels were low. Perhaps the overproduction of Prt1 altered interactions within a multifactor complex (MFC) that contained eIF3, eIF1, and eIF5 and was associated with TC $[38,39]$. One possible consequence of such a hypothetical rearrangement is the facilitation of TC recruitment despite its low level. Another possibility is that the Prt1 overdose affects the rearrangement of eIF3 at later stages of translation initiation. Structural studies revealed relocation of the Prt1-containing module upon mRNA binding, thus supporting a role for Prt1 in the interaction with the ribosome and selection of the AUG start codon [11,40-42]. Notably, the prt1-1 mutant was previously shown to affect the Gcn4 response by altering the stringency of AUG selection during ribosomal scanning on GCN4 mRNA [37]. The impairment in GCN4 translation in rpc128-1007 cells by Prt1 overdose suggests the stimulation of an as yet undefined step in the initiation of general translation machinery. Therefore, our hypothesis is that Prt1 indirectly suppresses the growth phenotype of the rpc128-1007 mutant by improving translation and growth despite low tRNA levels.

The enhancement of the Gcn4 response in rpc128-1007 cells by the overproduction of Rbs1 with an inactive $\mathrm{R} 3 \mathrm{H}$ domain (Figure 2D,E) is another interesting observation. Moreover, mRNA clustering indicated the enhancement of the effect of $r p c 128-1007$ on genes in clusters 1 and 2, indicating a dominant-negative effect of the [RBS1-R3H] plasmid. The dominant mode of the RBS1-R3H allele was not supported by simple genetic analysis of the rbs1 $\Delta$ strain (Supplementary Figure S1). The functional negative effect of the mutated Rbs1$\mathrm{R} 3 \mathrm{H}$ protein that was observed herein is unclear. Rbs1 is a poly(A) mRNA-binding protein. Mutational analysis indicated that the R3H domain is required for mRNA interactions [2]. Another RNA-binding domain, SUZ, was identified in the Rbs1 sequence, but cooperation between both the R3H and SUZ domains is still obscure. Therefore, one hypothesis is that the SUZ domain alone exerts a negative effect in Rbs1 that lacks a functional R3H domain. Specific effects of Rbs1 on mRNA expression could be explained by an interaction between Rbs1 and regulatory sequences in mRNA.

The decrease in tRNA levels in rpc128-1007 cells correlated with the downregulation of genes that are involved in protein degradation (Figure 2D), including 27 of the 31 subunits of the proteasome. Although the changes in expression were less than two-fold (Figure 2E, Supplementary Table S2), they represent a global tendency toward protein rescue. The coupling of tRNA biogenesis with protein degradation was also observed previously in yeast strains harboring deletions of single tRNA genes [28]. Deletions of tRNA genes representing the multicopy tRNA families led to downregulation of proteasomal gene expression; whereas deletions of single-copy tRNA genes have the opposite effect, i.e., upregulation. Additionally, single deletions of tRNA genes from the first group induced genes involved in amino acids metabolism and ribosome biosynthesis, but expression of these genes is decreased by deletions of tRNA genes from the second group [28]. We assume the equal transcription inhibition of all tRNA genes due to the Pol III assembly defect in $r p c 128-1007$ cells. However, the genome-wide effects of $r p c 128-1007$ mutation on mRNA expression look like those observed for deletions of tRNA genes from multicopy gene families but not for deletions of genes from single-copy gene families.

Finally, the examination of mRNA expression for individual genes using RT-qPCR (Figure 3) generally corroborated the RNAseq results; however, it revealed diverse genespecific effects. These may reflect the chromosomal locations of genes and the specific regulation of mRNA expression by Prt1 and Rbs1. 


\section{Materials and Methods}

\subsection{Strains, Plasmids, and Media}

The rpc128-1007 mutant MJ15-9C (MATa SUP11 ade2-1 ura3-1 lys2-1 leu2-3,112 his3) and wild-type strain MB159-4D (control) (MATa SUP11 ura3 leu2 ade2-1 his3 lys2-1) were described previously [43]. MB159-4D is referred to as wild type (wt) in Figure 1 and Figure S1.

The rpc128-1007 mutant that harbored the native RPC128 gene on the pRS316 centromeric plasmid pLH3 (kindly supplied by P. Thuriaux) was used as an isogenic control strain in the RNA-seq experiment and is referred to as wt in Figures 2 and 3, Figures S2 and S3, and Table S2.

The $r p c 128-1007 \Delta g c n 4$ strain was selected from the progeny of the cross of $r p c 128-1007$ mutant with the BY4741 $\Delta g c n 4$ strain (Euroscrarf, Germany, Oberursel).

IMT1 plasmid containing gene encoding tRNA ${ }_{i}{ }^{\text {Met }}$ cloned in Yep352 plasmid was provided by Oliver Lefebvre. YEp181-RBS1 and YEp181-RBS1-R3H plasmids, referred to herein as [RBS1] and [RBS1-R3H], respectively, were described previously [2]. pFL44L[URA3

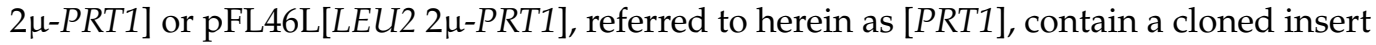
from chromosome $\mathrm{XV}$ originally selected in a screen of $r p c 128-1007$ suppressors [1]. This insert contains complete PRT1 and the $3^{\prime}$ part of the PRE10 gene. PRT1 was essential for suppression, demonstrated by deletion of the PmlI/Sall fragment of the insert. The p180 (GCN4-lacZ URA3 CEN) reporter plasmid was provided by L. Valasek [44].

The following media were used for growing yeast: YPD ( $2 \%$ glucose $(\mathrm{POCH}$, Gliwice, Poland, catalogue no. 459560117), 2\% peptone (Gibco, Thermo Fisher Scientific, Waltham, MA, USA, catalogue no. 211677), and $1 \%$ yeast extract (Gibco, catalogue no. 212750); and SC ( $2 \%$ glucose $(\mathrm{POCH}$, catalogue no. 459560117$)$ and $0.67 \%$ yeast nitrogen base without amino acids (Difco, Sparks, MD, USA, catalogue no. 0919-15). SC-ura, SC-leu, and SC-ura-leu contained $20 \mu \mathrm{g} / \mathrm{mL}$ of the amino acids that were required for growth, with the exception of uracil, leucine, or both, respectively.

\subsection{Analysis of GCN4-lacZ Expression}

Strains that were transformed with p180 (GCN4-LacZ URA3 CEN) were grown under nonstarvation conditions at a permissive temperature $\left(30^{\circ} \mathrm{C}\right)$ to an optical density (OD600) of 0.6 in standard rich YPD medium (yeast extract-peptone-dextrose) or under starvation conditions (growth in YPD before being moved to minimal SC medium with no amino acids for $3 \mathrm{~h}$ ). The cells were collected and protein extracts were prepared with a glass bead procedure [12]. $\beta$-galactosidase activity in whole-cell extracts was then measured as previously described [12].

\subsection{Northern Blot Analysis}

Overnight yeast cultures were resuspended in fresh YPD media to an OD600 of 0.2 and grown to the exponential phase $\left(\mathrm{OD}_{600}=0.6\right)$. The cultures were then shifted to $16{ }^{\circ} \mathrm{C}$, incubated for $2 \mathrm{~h}$, and harvested. RNA was extracted as described previously [2]. RNA $(5 \mu \mathrm{g})$ was separated by electrophoresis on a $10 \%$ polyacrylamide, $8 \mathrm{M}$ urea gel, transferred to positively charged nylon membranes, and hybridized with DIGlabeled probes as described previously [45]. Blots were developed using higher-resolution photographic films (Medical X-Ray film blue from AGFA, Morstel, Belgium). RNA signals were calculated using ImageJ software. The following DIG-labeled probes were applied: $\mathrm{tRNA}_{i} \mathrm{Met}^{\mathrm{MeAU})}$ (5'-TCGGTTTCGATCCGAGGACATCAGGGTTATGA-DIG-3'), tRNA $^{\text {Phe(GAA) }}\left(5^{\prime}\right.$-GCGCTCTCCCAACTGAGCT-DIG-3'), tRNA ${ }^{\text {Tyr(GUA) }} 5^{\prime}$-CGAGTCGAAC GCCCGAT-DIG-3' ${ }^{\prime}$, and 5.8S rRNA (5'-GCGTTGTTCATCGATGC-DIG-3').

\subsection{RNA Extraction and RNA-seq Procedure}

The original liquid cultures were grown overnight in SC-ura or SC-leu, transferred to $\mathrm{YPD}$, and grown to the log phase $(\mathrm{OD} 600=0.6)$. Total RNA was extracted from yeast cells using the Bead-beat Total RNA Mini kit (A\&A Biotechnology, Gdańsk, Poland, catalogue no. 031-25BB) and purified using Clean-Up RNA Concentrator (A\&A Biotechnology, 
catalogue no. 039-25C). RNA quality control was checked with Bioanalyzer 2100 (Agilent Technologies, Santa Clara, CA, USA).

RNA sequencing and data quality filtration were performed by the Hemispherian company with the following procedure: mRNA was enriched using oligo(dT) beads. The mRNA was fragmented randomly, and then cDNA was synthesized using an mRNA template and random hexamers primer, after which a custom second-strand synthesis buffer (Illumina), dNTPs, RNase H, and DNA Pol I were added to initiate second-strand synthesis. After a series of terminal repair and ligation of sequencing adaptors, the doublestranded cDNA library was completed through size selection and PCR enrichment.

Whole-transcriptome RNA-seq was performed using an Illumina NextSeq-500 sequencer (Novogene). Raw data that comprised three biological replicates for each strain were processed by removing reads that contained adapters and low-quality reads. The reads, having passed both operations, comprised $96 \%$ of the initial data, with an average read length of 150 bases.

\subsection{RNA-seq Data Quantification and Analysis}

The retained high-quality pair-end reads for each sample were mapped to the Saccharomyces cerevisiae genome sequences (version Saccharomyces_cerevisiae_EF4_Ensembl) using TopHat2 v 2.0.11 software on the genepattern website [46,47]. Quantitative analyses were performed using R version 3.6.3 software with the Bioconductor packages Genomic Alignments version 1.20.1 and DESeq2 version 1.24.0 [15-17].

The genes with adjusted values of $p<0.05$ were considered significantly differentially expressed. Fold changes (FCs) for these genes are marked in yellow in Supplementary Table S2. The principal component analysis (PCA) plot was performed using normalized reads in the DESeq2 package [16].

For the clustering analysis, we used a subset of genes with adjusted values of $p<0.05$. Fold changes for genes that did not pass filtration were changed to 0 . Hierarchical clustering was performed using the hclust algorithm with the Euclidean method in the R package. Next, we removed from the analysis genes that did not have significant changes in any of the comparisons analyzed. The data were divided into 10 groups using the k-means function with default parameters. For heatmap generation, we used the pheatmap version 1.0.12 package from $\mathrm{R}$.

To validate fold change between strains, we selected genes annotated in functional GO terms according to SGD (www.yeastgenome.org). Boxes in boxplots present data between first and third quartile ( $50 \%$ of observations), the line marks show median, and whiskers range between 5 th and 95 th (90\% observations) percentile. Statistical analysis was performed using Mann-Whitney test. The boxplots were generated using ggpubr version 0.3.0 (https:/ / github.com/kassambara/ggpubr, accessed on 31 May 2021).

\section{6. cDNA Synthesis and Reverse-Transcription Quantitative PCR (RT-qPCR)}

RNA (100 ng) was used for cDNA synthesis using the QuantiTect reverse transcriptase kit (Qiagen). cDNA for each sample was generated according to the manufacturer's instructions. The RT-qPCR reactions contained $1 \mu \mathrm{L}$ of cDNA template, $300 \mathrm{nM}$ primer pairs, and $5 \mu \mathrm{L}$ of RT-PCR Mix SYBR (A\&A Biotechnology). Quantitative PCR was performed using the Roche LightCycler 480 System with 5-min incubation at $95^{\circ} \mathrm{C}$, followed by 40 cycles at $95^{\circ} \mathrm{C}$ for $30 \mathrm{~s}, 6{ }^{\circ} \mathrm{C}$ for $20 \mathrm{~s}$, and $72{ }^{\circ} \mathrm{C}$ for $20 \mathrm{~s}$ (with a plate read after each cycle). A melting curve analysis was performed for each sample after PCR amplification to ensure that a single product with the expected melting curve characteristics was obtained. Each sample was loaded in triplicate. Each plate contained cDNA dilutions for the standard curve, a nonreverse transcriptase control, and a no template control. Polymerase chain reaction efficiencies were between $90 \%$ and $100 \%$. Data were processed using LightCycler 480 software and then analyzed in Microsoft Excel. Data are presented in arbitrary units, calculated from a standard curve, where the highest cDNA concentration was set to 1 . 
Values were normalized to the levels of $A C T 1$ mRNA encoding actin, which was used as an internal control. The primer sequences are listed in Supplementary Table S1.

Supplementary Materials: The following are available online at https:/ / www.mdpi.com/article/10 .3390/ijms22147298/s1.

Author Contributions: Conceptualization, M.B., I.R., T.W.T.; Investigation, I.R., M.C., A.A., E.L.; Methodology, I.R., M.C., E.L., A.A.; Software, I.R., T.W.T.; Validation and formal analysis, I.R., T.W.T., M.B., I.R., M.C., A.A., E.L.; Resources, M.C. and E.L.; Data curation, I.R.; Writing-original draft preparation, M.B.; Project administration, and funding acquisition, M.B. All authors have read and agreed to the published version of the manuscript.

Funding: This work was supported by National Science Centre (UMO-2017/25/B/NZ1/01889).

Institutional Review Board Statement: Not applicable.

Informed Consent Statement: Not applicable.

Data Availability Statement: RNA-seq data are available on Gene Expression Omnibus (https: / / www.ncbi.nlm.nih.gov/geo/, accessed on 31 May 2021). Accession number: GSE166918. Data were submitted on 17 February 2021.

Acknowledgments: We thank Andrzej Dziembowski and Anna Hojka-Osinska for their help in RNA-seq data analysis.

Conflicts of Interest: The authors declare no conflict of interest.

\section{References}

1. Cieśla, M.; Makała, E.; Płonka, M.; Bazan, R.; Gewartowski, K.; Dziembowski, A.; Boguta, M. Rbs1, a New Protein Implicated in RNA Polymerase III Biogenesis in Yeast Saccharomyces Cerevisiae. Mol. Cell. Biol. 2015, 35, 1169-1181. [CrossRef]

2. Cieśla, M.; Turowski, T.W.; Nowotny, M.; Tollervey, D.; Boguta, M. The Expression of Rpb10, a Small Subunit Common to RNA Polymerases, Is Modulated by the R3H Domain-Containing Rbs1 Protein and the Upf1 Helicase. Nucleic Acids Res. 2020, 48, 12252-12268. [CrossRef]

3. Wild, T.; Cramer, P. Biogenesis of Multisubunit RNA Polymerases. Trends Biochem. Sci. 2012, 37, 99-105. [CrossRef]

4. Ramsay, E.P.; Abascal-Palacios, G.; Daiß, J.L.; King, H.; Gouge, J.; Pilsl, M.; Beuron, F.; Morris, E.; Gunkel, P.; Engel, C.; et al. Structure of Human RNA Polymerase III. Nat. Commun. 2020, 11. [CrossRef]

5. Girbig, M.; Misiaszek, A.D.; Vorländer, M.K.; Lafita, A.; Grötsch, H.; Baudin, F.; Bateman, A.; Müller, C.W. Cryo-EM Structures of Human RNA Polymerase III in Its Unbound and Transcribing States. Nat. Struct. Mol. Biol. 2021, 28, 210-219. [CrossRef] [PubMed]

6. Wang, Q.; Li, S.; Wan, F.; Xu, Y.; Wu, Z.; Cao, M.; Lan, P.; Lei, M.; Wu, J. Structural Insights into Transcriptional Regulation of Human RNA Polymerase III. Nat. Struct. Mol. Biol. 2021, 28, 220-227. [CrossRef] [PubMed]

7. Gadal, O.; Shpakovski, G.V.; Thuriaux, P. Mutants in ABC10beta, a Conserved Subunit Shared by All Three Yeast RNA Polymerases, Specifically Affect RNA Polymerase I Assembly. J. Biol. Chem. 1999, 274, 8421-8427. [CrossRef] [PubMed]

8. Cieśla, M.; Mierzejewska, J.; Adamczyk, M.; Farrants, A.-K.Ö.; Boguta, M. Fructose Bisphosphate Aldolase Is Involved in the Control of RNA Polymerase III-Directed Transcription. Biochim. Biophys. Acta 2014, 1843, 1103-1110. [CrossRef] [PubMed]

9. Turowski, T.W.; Boguta, M. Specific Features of RNA Polymerases I and III: Structure and Assembly. Front. Mol. Biosci. 2021, 8. [CrossRef] [PubMed]

10. Phan, L.; Zhang, X.; Asano, K.; Anderson, J.; Vornlocher, H.P.; Greenberg, J.R.; Qin, J.; Hinnebusch, A.G. Identification of a Translation Initiation Factor 3 (EIF3) Core Complex, Conserved in Yeast and Mammals, That Interacts with EIF5. Mol. Cell. Biol. 1998, 18, 4935-4946. [CrossRef]

11. Zeman, J.; Itoh, Y.; Kukačka, Z.; Rosůlek, M.; Kavan, D.; Kouba, T.; Jansen, M.E.; Mohammad, M.P.; Novák, P.; Valášek, L.S. Binding of EIF3 in Complex with EIF5 and EIF1 to the 40S Ribosomal Subunit Is Accompanied by Dramatic Structural Changes. Nucleic Acids Res. 2019, 47, 8282-8300. [CrossRef] [PubMed]

12. Conesa, C.; Ruotolo, R.; Soularue, P.; Simms, T.A.; Donze, D.; Sentenac, A.; Dieci, G. Modulation of Yeast Genome Expression in Response to Defective RNA Polymerase III-Dependent Transcription. Mol. Cell. Biol. 2005, 25, 8631-8642. [CrossRef] [PubMed]

13. Hinnebusch, A.G.; Natarajan, K. Gcn4p, a Master Regulator of Gene Expression, Is Controlled at Multiple Levels by Diverse Signals of Starvation and Stress. Eukaryot. Cell 2002, 1, 22-32. [CrossRef] [PubMed]

14. Archambault, J.; Friesen, J.D. Genetics of Eukaryotic RNA Polymerases I, II, and III. Microbiol. Rev. 1993, 57, 703-724. [CrossRef] [PubMed]

15. Lawrence, M.; Huber, W.; Pagès, H.; Aboyoun, P.; Carlson, M.; Gentleman, R.; Morgan, M.T.; Carey, V.J. Software for Computing and Annotating Genomic Ranges. PLoS Comput. Biol. 2013, 9, e1003118. [CrossRef] 
16. Love, M.I.; Huber, W.; Anders, S. Moderated Estimation of Fold Change and Dispersion for RNA-Seq Data with DESeq2. Genome Biol. 2014, 15, 550. [CrossRef] [PubMed]

17. Love, M.I.; Anders, S.; Kim, V.; Huber, W. RNA-Seq Workflow: Gene-Level Exploratory Analysis and Differential Expression. F1000Research 2015, 4, 1070. [CrossRef]

18. Natarajan, K.; Meyer, M.R.; Jackson, B.M.; Slade, D.; Roberts, C.; Hinnebusch, A.G.; Marton, M.J. Transcriptional Profiling Shows That Gcn4p Is a Master Regulator of Gene Expression during Amino Acid Starvation in Yeast. Mol. Cell. Biol. 2001, 21, 4347-4368. [CrossRef]

19. Rawal, Y.; Chereji, R.V.; Valabhoju, V.; Qiu, H.; Ocampo, J.; Clark, D.J.; Hinnebusch, A.G. Gcn4 Binding in Coding Regions Can Activate Internal and Canonical 5' Promoters in Yeast. Mol. Cell 2018, 70, 297-311.e4. [CrossRef]

20. Barski, A.; Chepelev, I.; Liko, D.; Cuddapah, S.; Fleming, A.B.; Birch, J.; Cui, K.; White, R.J.; Zhao, K. Pol II and Its Associated Epigenetic Marks Are Present at Pol III-Transcribed Non-Coding RNA Genes. Nat. Struct. Mol. Biol. 2010, 17, 629-634. [CrossRef] [PubMed]

21. Canella, D.; Bernasconi, D.; Gilardi, F.; LeMartelot, G.; Migliavacca, E.; Praz, V.; Cousin, P.; Delorenzi, M.; Hernandez, N.; Consortium, T.C.; et al. A Multiplicity of Factors Contributes to Selective RNA Polymerase III Occupancy of a Subset of RNA Polymerase III Genes in Mouse Liver. Genome Res. 2012, 22, 666-680. [CrossRef]

22. Raha, D.; Wang, Z.; Moqtaderi, Z.; Wu, L.; Zhong, G.; Gerstein, M.; Struhl, K.; Snyder, M. Close Association of RNA Polymerase II and Many Transcription Factors with Pol III Genes. Proc. Natl. Acad. Sci. USA 2010, 107, 3639-3644. [CrossRef] [PubMed]

23. Moqtaderi, Z.; Wang, J.; Raha, D.; White, R.J.; Snyder, M.; Weng, Z.; Struhl, K. Genomic Binding Profiles of Functionally Distinct RNA Polymerase III Transcription Complexes in Human Cells. Nat. Struct. Mol. Biol. 2010, 17, 635-640. [CrossRef]

24. Oler, A.J.; Alla, R.K.; Roberts, D.N.; Wong, A.; Hollenhorst, P.C.; Chandler, K.J.; Cassiday, P.A.; Nelson, C.A.; Hagedorn, C.H.; Graves, B.J.; et al. Human RNA Polymerase III Transcriptomes and Relationships to Pol II Promoter Chromatin and Enhancer-Binding Factors. Nat. Struct. Mol. Biol. 2010, 17, 620-628. [CrossRef]

25. Yeganeh, M.; Praz, V.; Cousin, P.; Hernandez, N. Transcriptional Interference by RNA Polymerase III Affects Expression of the Polr3e Gene. Genes Dev. 2017, 31, 413-421. [CrossRef] [PubMed]

26. Wang, Q.; Nowak, C.M.; Korde, A.; Oh, D.-H.; Dassanayake, M.; Donze, D. Compromised RNA Polymerase III Complex Assembly Leads to Local Alterations of Intergenic RNA Polymerase II Transcription in Saccharomyces Cerevisiae. BMC Biol. 2014, 12, 89. [CrossRef] [PubMed]

27. Gerber, A.; Ito, K.; Chu, C.-S.; Roeder, R.G. Gene-Specific Control of TRNA Expression by RNA Polymerase II. Mol. Cell 2020, 78, 765-778.e7. [CrossRef] [PubMed]

28. Bloom-Ackermann, Z.; Navon, S.; Gingold, H.; Towers, R.; Pilpel, Y.; Dahan, O. A Comprehensive TRNA Deletion Library Unravels the Genetic Architecture of the TRNA Pool. PLoS Genet. 2014, 10, e1004084. [CrossRef] [PubMed]

29. Hinnebusch, A.G. Translational Regulation of GCN4 and the General Amino Acid Control of Yeast. Annu. Rev. Microbiol. 2005, 59, 407-450. [CrossRef]

30. Jia, M.H.; Larossa, R.A.; Lee, J.-M.; Rafalski, A.; Derose, E.; Gonye, G.; Xue, Z. Global Expression Profiling of Yeast Treated with an Inhibitor of Amino Acid Biosynthesis, Sulfometuron Methyl. Physiol. Genom. 2000, 3, 83-92. [CrossRef] [PubMed]

31. Patil, C.K.; Li, H.; Walter, P. Gcn4p and Novel Upstream Activating Sequences Regulate Targets of the Unfolded Protein Response. PLoS Biol. 2004, 2, E246. [CrossRef] [PubMed]

32. Han, L.; Guy, M.P.; Kon, Y.; Phizicky, E.M. Lack of 2'-O-Methylation in the TRNA Anticodon Loop of Two Phylogenetically Distant Yeast Species Activates the General Amino Acid Control Pathway. PLoS Genet. 2018, 14, e1007288. [CrossRef] [PubMed]

33. Ghavidel, A.; Kislinger, T.; Pogoutse, O.; Sopko, R.; Jurisica, I.; Emili, A. Impaired TRNA Nuclear Export Links DNA Damage and Cell-Cycle Checkpoint. Cell 2007, 131, 915-926. [CrossRef]

34. Qiu, H.; Hu, C.; Anderson, J.; Björk, G.R.; Sarkar, S.; Hopper, A.K.; Hinnebusch, A.G. Defects in TRNA Processing and Nuclear Export Induce GCN4 Translation Independently of Phosphorylation of the Alpha Subunit of Eukaryotic Translation Initiation Factor 2. Mol. Cell. Biol. 2000, 20, 2505-2516. [CrossRef] [PubMed]

35. Steffen, K.K.; MacKay, V.L.; Kerr, E.O.; Tsuchiya, M.; Hu, D.; Fox, L.A.; Dang, N.; Johnston, E.D.; Oakes, J.A.; Tchao, B.N.; et al Yeast Life Span Extension by Depletion of 60s Ribosomal Subunits Is Mediated by Gcn4. Cell 2008, 133, 292-302. [CrossRef]

36. Danaie, P.; Wittmer, B.; Altmann, M.; Trachsel, H. Isolation of a Protein Complex Containing Translation Initiation Factor Prt1 from Saccharomyces Cerevisiae. J. Biol. Chem. 1995, 270, 4288-4292. [CrossRef]

37. Nielsen, K.H.; Szamecz, B.; Valásek, L.; Jivotovskaya, A.; Shin, B.-S.; Hinnebusch, A.G. Functions of EIF3 Downstream of 48S Assembly Impact AUG Recognition and GCN4 Translational Control. EMBO J. 2004, 23, 1166-1177. [CrossRef]

38. Asano, K.; Clayton, J.; Shalev, A.; Hinnebusch, A.G. A Multifactor Complex of Eukaryotic Initiation Factors, EIF1, EIF2, EIF3, EIF5, and Initiator TRNA(Met) Is an Important Translation Initiation Intermediate in Vivo. Genes Dev. 2000, 14, 2534-2546. [CrossRef]

39. Wagner, S.; Herrmannová, A.; Hronová, V.; Gunišová, S.; Sen, N.D.; Hannan, R.D.; Hinnebusch, A.G.; Shirokikh, N.E.; Preiss, T.; Valášek, L.S. Selective Translation Complex Profiling Reveals Staged Initiation and Co-Translational Assembly of Initiation Factor Complexes. Mol. Cell 2020, 79, 546-560.e7. [CrossRef]

40. Chiu, W.-L.; Wagner, S.; Herrmannová, A.; Burela, L.; Zhang, F.; Saini, A.K.; Valásek, L.; Hinnebusch, A.G. The C-Terminal Region of Eukaryotic Translation Initiation Factor 3a (EIF3a) Promotes MRNA Recruitment, Scanning, and, Together with EIF3j and the EIF3b RNA Recognition Motif, Selection of AUG Start Codons. Mol. Cell. Biol. 2010, 30, 4415-4434. [CrossRef] 
41. Elantak, L.; Wagner, S.; Herrmannová, A.; Karásková, M.; Rutkai, E.; Lukavsky, P.J.; Valásek, L. The Indispensable N-Terminal Half of EIF3j/HCR1 Cooperates with Its Structurally Conserved Binding Partner EIF3b/PRT1-RRM and with EIF1A in Stringent AUG Selection. J. Mol. Biol. 2010, 396, 1097-1116. [CrossRef] [PubMed]

42. Simonetti, A.; Brito Querido, J.; Myasnikov, A.G.; Mancera-Martinez, E.; Renaud, A.; Kuhn, L.; Hashem, Y. EIF3 Peripheral Subunits Rearrangement after MRNA Binding and Start-Codon Recognition. Mol. Cell 2016, 63, 206-217. [CrossRef]

43. Cieśla, M.; Towpik, J.; Graczyk, D.; Oficjalska-Pham, D.; Harismendy, O.; Suleau, A.; Balicki, K.; Conesa, C.; Lefebvre, O.; Boguta, M. Maf1 Is Involved in Coupling Carbon Metabolism to RNA Polymerase III Transcription. Mol. Cell. Biol. 2007, 27, 7693-7702. [CrossRef] [PubMed]

44. Hinnebusch, A.G. A Hierarchy of Trans-Acting Factors Modulates Translation of an Activator of Amino Acid Biosynthetic Genes in Saccharomyces Cerevisiae. Mol. Cell. Biol. 1985, 5, 2349-2360. [CrossRef]

45. Wu, J.; Huang, H.-Y.; Hopper, A.K. A Rapid and Sensitive Non-Radioactive Method Applicable for Genome-Wide Analysis of Saccharomyces Cerevisiae Genes Involved in Small RNA Biology. Yeast Chichester Engl. 2013, 30, 119-128. [CrossRef] [PubMed]

46. Reich, M.; Liefeld, T.; Gould, J.; Lerner, J.; Tamayo, P.; Mesirov, J.P. GenePattern 2.0. Nat. Genet. 2006, 38, 500-501. [CrossRef] [PubMed]

47. Trapnell, C.; Roberts, A.; Goff, L.; Pertea, G.; Kim, D.; Kelley, D.R.; Pimentel, H.; Salzberg, S.L.; Rinn, J.L.; Pachter, L. Differential Gene and Transcript Expression Analysis of RNA-Seq Experiments with TopHat and Cufflinks. Nat. Protoc. 2012, 7, 562-578. [CrossRef] 